\title{
Pressure from data-driven-estimated velocity fields using snapshot PIV and fast probes
}

\author{
Marco Raiola $^{1 *}$, Junwei Chen ${ }^{1}$, Stefano Discetti ${ }^{1}$ \\ ${ }^{1}$ Universidad Carlos III de Madrid, Aerospace Engineering Research Group, Madrid, Spain \\ *mraiola@ing.uc3m.es
}

\begin{abstract}
This work explores the use of data-driven techniques to retrieve time-resolved information from snapshot PIV by exploiting the information from synchronized high-repetition rate sensors measuring flow quantities in few points, and to compute from it the instantaneous pressure field leveraging the Navier-Stokes momentum equation of the flow. This work focus on a technique rooted in the Extended Proper Orthogonal Decomposition, which already proven good performances in estimating time-resolved velocity fields from a finite number of probes synchronized with field measurements. The performances of the technique and its robustness to noise are tested on 2 synthetic dataset, a laminar one and a turbulent one, and compared to the most commonly applied technique to retrieve time-resolved information from snapshot PIV which exploits Taylor's hypothesis.
\end{abstract}

\section{Introduction}

The availability of three-dimensional three-components (3D-3C) velocity fields, with time-resolution (thus 4D-3C) has open the path in the last decade to the measurements of pressure fields (Van Oudheusden, 2013; Van Gent et al., 2017). This is normally achieved enforcing the validity of the momentum equation:

$$
\nabla p=-\rho \frac{D \mathbf{u}}{D t}+\mu \nabla^{2} \mathbf{u}=\mathbf{f}(\mathbf{u})
$$

where $\mu$ is the fluid dynamic viscosity, $D \mathbf{u} / D t$ is the Lagrangian acceleration, and $\nabla$ is the gradient operator. When the full 3D velocity and acceleration (either Eulerian or Lagrangian) is available, the above equation, or equivalent formulations derived from it, enables the estimation of 3D instantaneous pressure fields. Such information can be exploited, for instance, for the study of unsteady pressure forces on surfaces or to locate noise source. This approach is possible for low-speed flows, for which hardware for time-resolved measurement is available, thus allowing to directly measure $D \mathbf{u} / D t$. For medium/high Reynolds number measurements, where time-resolution is often not available, the pressure can be obtained only under strong assumptions, such as selection of the magnitude of the convection velocity (Van der Kindere et al., 2019) or non-dissipative advection of vortices (Schneiders et al., 2018).

A promising approach to obtain time-resolution from standard low-repetition-rate equipment is the combination of PIV with fast point probes located strategically in the flow field. Statistical evidence of correlation between probe and field measurements can be enforced to train the probes to estimate flow fields. One popular solution is based on Extended Proper Orthogonal Decomposition (EPOD, Borée 2003), which relates the most relevant features observed on synchronized measurements of flow fields and probe data via modal analysis. The method was implemented and successfully applied in the past years (Tinney et al., 2008; Hosseini et al., 2015), with particular success in flows with dominant frequencies which allow a compact representation with only few relevant modes. Discetti et al. (2018) proposed an implementation including a robust filtering, which enabled the application to spectrally-rich turbulent flows and demonstrated its feasibility in an unprecedented high-Reynolds-number pipe-flow experiment in the CICLOPE facility (Discetti et al., 2019).

In this work we combine simultaneous non-time-resolved field measurements and time-resolved pointwise measurements to achieve the time resolution needed to obtain pressure fields from the integration of 
the momentum equation. The field estimation is carried out using EPOD and the virtual probes method (Hosseini et al., 2015; Discetti et al., 2018). The method consists in enforcing the correlation between field and probe data by including for each snapshots the data recorded by the probe within a time segment (i.e. not only at the same instant the snapshot was recorded). For convective flows, this is equivalent to have an additional set of virtual probes, thus making available more information to establish the correlation between field and probe data. Whether the reconstructed fields are of sufficient quality to be used for pressure estimation from Eq. 1, it is still an unexplored field. The method is tested using a DNS-database of the wake of three cylinders in a configuration referred as fluidic pinball (Deng et al., 2020) and a DNS-database of a turbulent channel flow from the Johns Hopkins Turbulence Database (JHTDB, Li et al., 2008).

\section{EPOD-based estimation of time-resolved velocity fields}

POD (Proper Orthogonal Decomposition) is an unsupervised learning data-driven method to obtain modal decomposition of datasets. This method splits the velocity field data into the weighted sum of modes (both temporal and spatial) orthogonal to each other and arranged by decreasing energy content.

Assume that $n_{t}$ snapshots of the fluctuating velocity field are arranged into a matrix $\mathrm{U}$, where each row (with length $n_{p}$ ) contains the velocity components in all the nodes of the domain. The matrix $U$ (of size $n_{t} \times n_{p}$, where typically $n_{t} \leq n_{p}$ ) can be decomposed by the economy-size Singular Value Decomposition (SVD), i.e.

$$
U=\Psi \Sigma \Phi^{T}
$$

In the decomposition of Eq. 2, the columns of the $n_{t} \times n_{t}$ orthogonal matrix $\Psi$ contains the temporal modes $\psi_{i}$, the columns of the $n_{t} \times n_{p}$ orthogonal matrix $\Phi^{T}$ contains the spatial modes $\phi_{i}, \Sigma$ is a square diagonal matrix containing the singular values $\sigma_{i}$ arranged in a decreasing order.

The same decomposition can be applied on the probe snapshot matrix $U_{p r}$ containing velocity data from $s$ high-frequency probes synchronized with velocity field measurements. In order to increase the quantity of probe data available for each snapshot, the virtual probe approach (Sicot et al., 2012; Hosseini et al., 2015) is employed: for each physical probe, a time-resolved sequence of $q$ probe samples is extracted after the velocity field sampling time and considered as additional probes under the Taylor's hypothesis. This results in a matrix $U_{p r}$ with $n_{t}$ rows (as the velocity field snapshots) and $n_{t t}=s \times q$ columns which can be decomposed as

$$
U_{p r}=\Psi_{p r} \Sigma_{p r} \Phi_{p r}^{T}
$$

For the problem under investigation, the extended POD modes $\Phi_{e}$ corresponding to the field measurements can be estimated as

$$
\Psi_{p r}^{T} U=\Sigma_{e} \Phi_{e}^{T}=\Phi_{p r}^{T} \Psi \Sigma \Phi^{T}=\Xi \Sigma \Phi^{T}
$$

where the subscript $e$ refers to extended POD modes and the matrix $\Xi=\Psi_{p r}^{T} \Psi$ is a matrix containing the information about the temporal correlation between field and probe modes.

Knowing the POD spatial modes $\left(\Phi\right.$ and $\left.\Phi_{p r}\right)$ and singular values $\left(\Sigma\right.$ and $\left.\Sigma_{p r}\right)$ of the velocity field and of the probe snapshot matrix, as well as the temporal correlations matrix $\Xi$, it is possible to estimate the velocity field $u_{e s t}$ at an off-sample instant from a probe data snapshot $u_{s e}$ sampled at that instant:

$$
u_{e s t}=u_{s e} \Phi_{p r} \Sigma_{p r}^{-1} \Xi \Sigma \Phi^{T}=\psi_{e s t} \Sigma \Phi^{T}
$$

As shown by Eq. 5, the estimation of the velocity field depends on all the probe modes through the matrix $\Xi$, accounting also for mode interaction. While this approach removes the need for multiple-time delays (used for instance in multi-time delay linear stochastic estimation), it also might result in a contamination of the estimation from spurious correlations between flow field and probe modes. To this purpose, Discetti et al. (2018) proposed to filter out low-correlation entries from the matrix $\Xi$ leveraging on the consideration that uncorrelated random modes might still produce a non-null random entry in $\Xi$ with a standard deviation equal to $n_{t}^{-0.5}$. The entries $\Xi_{i j}$ in the matrix $\Xi$ are therefore truncated following the 3 -sigma rule

$$
\Xi_{i j}=0, \text { when }-\frac{3}{\sqrt{n_{t}}} \leq \Xi_{i j} \leq \frac{3}{\sqrt{n_{t}}}, i, j=1,2, \ldots, n
$$




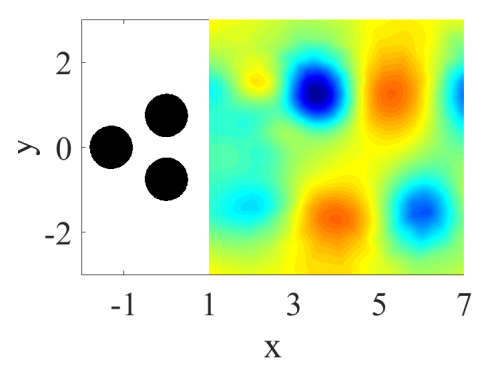

(a)

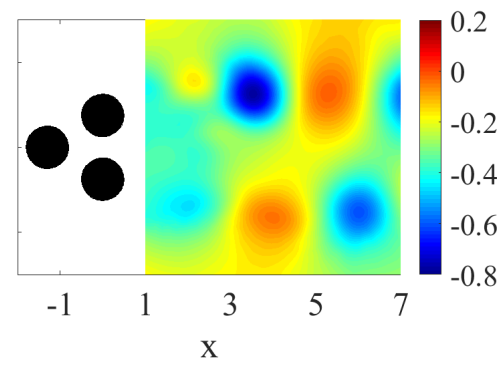

(b)

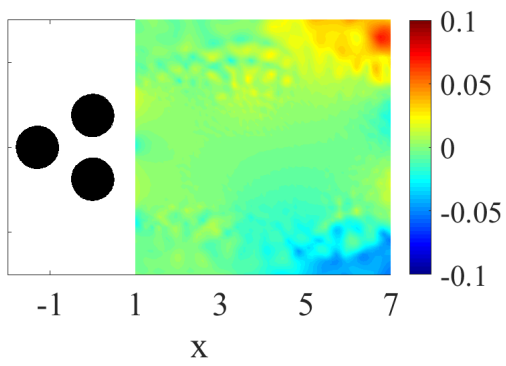

(c)

Figure 1: (a) pressure from DNS, (b) pressure estimated using iterative method, (c) error of pressure estimation.

which, on the hypothesis that the spurious-correlation entries follow a normal distribution, guarantees that $99.7 \%$ of them are removed. While this approach is extremely robust to noise, it might also cut out a small portion of the actual correlation between probes and flow fields. This approach will be referred to as $\Xi$-filtered approach in the reminder of the paper.

The need of filtering the estimation is especially relevant for pressure reconstruction, since spurious correlation might produce unacceptable levels of noise on the estimated fields, which are later amplified by the time derivative. Other alternatives for the filtering are based on the time-filtering of the estimated temporal mode $\psi_{\text {est }}$.In the present work a $6^{\text {th }}$ order low-pass Butterworth filter with cutoff frequency of 0.05 times of data sample rate is applied to the coefficient of every mode. This approach will be referred to as $\psi$-filtered approach in the reminder of the paper.

\section{Pressure integration algorithm from velocity field data}

Since the time-resolved fields to compute the Lagrangian acceleration are obtained through a process of estimation from point probes, it is possible that noise is amplified in the process. For this reason, a technique with high robustness to noise is implemented for integration of the pressure gradient. The pressure is estimated by integrating Eq. 1 using a finite-differences version of the Modified Richardson Iteration method (Richardson, 1910). For each point $\mathbf{x}_{m}$ in the domain, the iteration uses the value in the surrounding points $\mathbf{x}_{n}$ to refresh it, enforcing the pressure gradient $\nabla p=\mathbf{f}(\mathbf{u})$ from the momentum equation

$$
\begin{aligned}
p^{i+1}\left(\mathbf{x}_{m}\right) & =\frac{1}{N} \sum_{n=1}^{N}\left(p^{i}\left(\mathbf{x}_{n}\right)+\left.\mathbf{f}(\mathbf{u})\right|_{\mathbf{x}_{n}^{\prime}} \cdot\left(\mathbf{x}_{m}-\mathbf{x}_{n}\right)\right) \\
& =p^{i}\left(\mathbf{x}_{m}\right)+\frac{1}{N} \sum_{n=1}^{N}\left(\left.\mathbf{f}(\mathbf{u})\right|_{x_{n}^{\prime}} \cdot\left(\mathbf{x}_{m}-\mathbf{x}_{n}\right)-\frac{p^{i}\left(\mathbf{x}_{m}\right)-p^{i}\left(\mathbf{x}_{n}\right)}{\left|\mathbf{x}_{m}-\mathbf{x}_{n}\right|}\left(\left|\mathbf{x}_{m}-\mathbf{x}_{n}\right|\right)\right) \\
& =p^{i}\left(\mathbf{x}_{m}\right)+\frac{1}{N} \sum_{n=1}^{N}\left(\left.\mathbf{f}(\mathbf{u})\right|_{\mathbf{x}_{n}^{\prime}}-\left.\nabla p^{i}\right|_{\mathbf{x}_{n}^{\prime}}\right) \cdot\left(\mathbf{x}_{m}-\mathbf{x}_{n}\right)
\end{aligned}
$$

where $\mathrm{N}$ is the number of neighbouring points (for a Cartesian grid, 4 in $2 \mathrm{D}, 6$ in 3D), the gradients are calculated in the middle point $\mathbf{x}_{n}^{\prime}=\left(\mathbf{x}_{m}+\mathbf{x}_{n}\right) / 2$ to reduce truncation error, and $i$ is the index of iteration. A relaxation parameter $\omega<1 / N$ is introduced into Eq. 7, which becomes

$$
p^{i+1}\left(\mathbf{x}_{m}\right)=p^{i}\left(\mathbf{x}_{m}\right)+\omega \sum_{n=1}^{N}\left(\left.\mathbf{f}(\mathbf{u})\right|_{\mathbf{x}_{n}^{\prime}}-\left.\nabla p^{i}\right|_{\mathbf{x}_{n}^{\prime}}\right) \cdot\left(\mathbf{x}_{m}-\mathbf{x}_{n}\right)
$$

Points outside the domain are excluded from the computation in Eq. 8 when $\mathbf{x}_{m}$ is near the boundary, thus reducing $N$. The iteration is initialized with $p^{0}=\mathbf{0}$, updates globally, and interrupts when the difference between the pressure in two loops is below a threshold $\varepsilon$, i.e. when $\left\|p^{i+1}-p^{i}\right\|_{2}<\varepsilon$, where $\|\cdot\|_{2}$ is the 


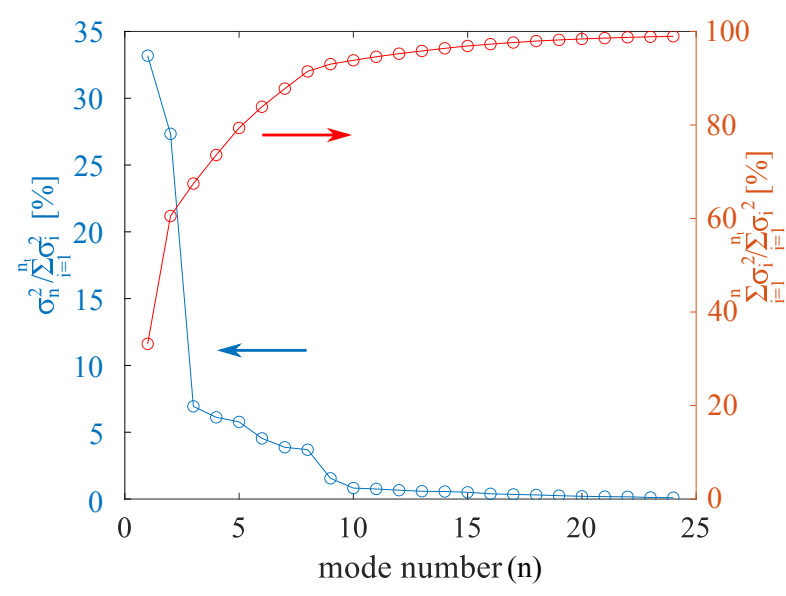

(a)

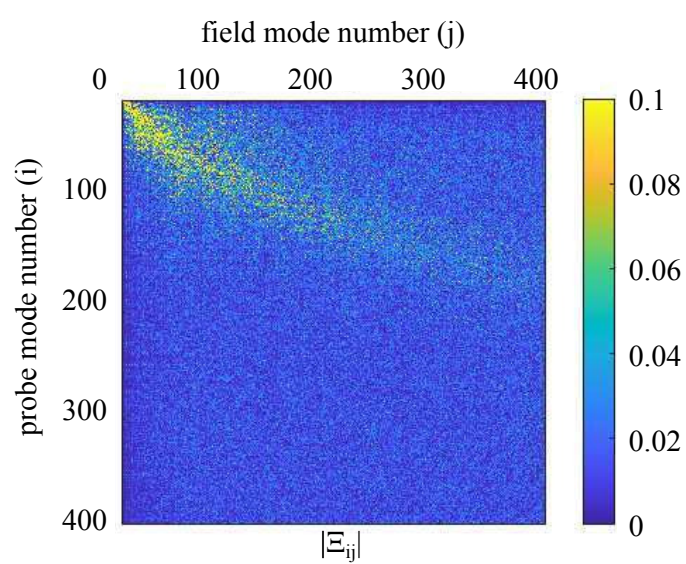

(b)

Figure 2: (a) Kinetic energy (blue) and cumulative kinetic energy (red) contained in the first 24 POD modes of flow field; (b) absolute value of $\Xi$ for the fluidic pinball test case.

$\mathcal{L}^{2}$ distance over all the points of the domain and the threshold $\varepsilon=10^{-5}$ is determined accordingly to the maximum accuracy of pressure that can be attained depending on velocity field data precision.

Iterative methods have been used in pressure estimation before by Tronchin et al. (2015). In their algorithm, the pressure value in $\mathbf{x}_{m}$ is updated using the first row of Eq. 7, i.e. only using of the pressure in the surrounding points and the gradient, without including the value of pressure in $\mathbf{x}_{m}$. This causes it harder to converge when the noise or error in the pressure gradient is significant. Besides, the global update in this paper makes the iteration result not rely to typical integration direction.

The performances of the iterative integration method described above are shown for a single snapshot of the fluidic pinball DNS database in Fig. 1. The dataset will be described in the following section. The estimation error is very low in most of the domain except for the top-right and bottom-right corner, where the DNS grid is more sparse and the truncation error is not negligible when the data are interpolated from the DNS grid to the Cartesian grid.

\section{Validation}

\subsection{Fluidic pinball}

The method is applied to synthetic data to assess the performance of the EPOD-based estimation of both velocity and pressure fields. The first synthetic dataset has been extracted from a 2D-DNS of the wake of a fluidic pinball (Deng et al., 2020). The simulation features three cylinders with radius $R=0.5$ standing in the domain, whose centres form an equilateral triangle with side length equal to $3 R$. One cylinder is located upstream while the other two are abreast, downstream with respect to the first one, see Fig. 1 . The two-dimensional DNS is performed at Re $=130$ (referred as chaotic regime, Deng et al., 2020).

The region selected to test the EPOD-based estimation is placed in the wake of the obstacles, ranging from $x=1$ to $x=7$ and from $y=-3$ to $y=3$. The velocity data are interpolated on a Cartesian grid with distance between two adjacent points of 0.08 in order to simulate the results of a PIV experiment. Five point probes measuring the 2 inplane components of velocity, each recording 60 samples-per-frame at a sample rate of 1/0.08, are placed at the downstream edge of the region $(x=7)$, with a spacing of 1 in the $y$ direction. The training dataset is composed of 4685 velocity field snapshots as well as of the synchronized virtual probe data, forming the matrices $U$ and $U_{p r}$, respectively, as reported in $\S 2$. The snapshots are selected to have a temporal spacing of 0.88 .

Fig. 2a reports the energy distribution of the POD modes $\left(\lambda_{i}=\sigma_{i}^{2}\right)$ with blue line, as well as the accumulated energy distribution with red line, after normalization with the total energy. The first few POD modes contain most of energy, over $95 \%$ of the total energy are in the first 12 modes.

Fig. $2 b$ shows the absolute value of the upper-left portion of unfiltered matrix $\Xi$, which is representative of the correlation between the $i^{\text {th }}$ temporal mode of probes and the $j^{\text {th }}$ temporal mode of flow field, where the 
$i$ and $j$ is the number of row and column in $\Xi$. The matrix $\Xi$ shows a clear diagonal dominance for at least the first 100 modes, which indicates an almost biunivocal correspondence between probe and field modes. As the number of mode is increased this dominance disappears and each probe mode tends to correspond to a larger number of field modes.

The performances of the $\psi$-filtered EPOD estimation are reported in Fig. 3 for a single snapshot not included in the training dataset, both in terms of flow field (Fig. 3a-b) and in terms of pressure (Fig. 3c). The DNS data are reported for comparison (Fig. 3a-c) as well as the error of the estimation with respect to the DNS (Fig. 3f-h). The error mainly affects the streamwise velocity component in the wake region (from $x=2$ to $x=4$ ), producing an error pattern which suggests the misplacement of flow structures in the $y$-direction. The crosswise velocity component shows lower levels of error, still localized in the same region. The localization of the reconstruction error might be an effect of the presence of intense small-scale flow features, strongly subjected to stretching and deformation. Additionally, this region stands farther from the probes. These two aspects contribute in the reduced capability of the probes to sense accurately the fluctuations in this region. Despite the presence of the error on the velocity field, the pressure reconstruction is affected by considerably lower levels of estimation error, suggesting that the integration is smoothing down the error in the spatial derivatives.

A more precise assessment of the estimation error has been carried out using the RMS of the error with respect to the DNS over 1500 estimated snapshots not included in the EPOD training dataset and which constitutes the testing dataset. The assessment includes also the estimation through the Taylor's hypothesis (TH), which is, to date, the most common option to estimate time-derivatives from snapshot PIV (Van der Kindere et al., 2019). Taylor's hypothesis relies on the assumption that the flow field fluctuations are advected with a velocity corresponding to the ensemble-averaged flow field $\overline{\mathbf{u}}$, i.e. $\partial \mathbf{u}^{\prime} / \partial t=-(\overline{\mathbf{u}} \cdot \nabla) \mathbf{u}^{\prime}$. This enables the estimation of the time derivative of velocity, and thus of the pressure, for a measured snapshot. Additionally, the TH can be used to propagate the measured frame in time, obtaining an estimated time-resolved sequence of velocity fields. The simplest way to fulfill it is by means of a unidirectional Euler propagation, which is

$$
\mathbf{u}\left(t_{0}+d t\right)=\mathbf{u}\left(t_{0}\right)+\left.\frac{\partial u}{\partial t}\right|_{t_{0}} d t=\mathbf{u}\left(t_{0}\right)-(\overline{\mathbf{u}} \cdot \nabla) \mathbf{u}^{\prime}\left(t_{0}\right) d t
$$

In the present case, the time-resolved sequence has been estimated with TH using a $4^{\text {th }}$ order Runge-Kutta method.

Fig. 4 shows the RMS estimation error for the unfiltered EPOD estimation (Fig. 4a-c), the $\Xi$-filtered EPOD estimation (Fig. 4d-f), the $\psi$-filtered EPOD (Fig. 4g-i) and the estimation using the Taylor's hypothesis (TH). Fig. 4 reports the error on the streamwise component of velocity on the left column (Fig. 4a,d,g), the error on the time-derivative on the streamwise component of velocity in the central column (Fig. 4b,e,h,j) and the error on pressure in the right column (Fig. 4c,f,i,k). For the TH, the estimation has been carried out in the least error conditions, i.e. assuming that the exact velocity field was available at the selected snapshot, thus using the TH only to estimate the time derivative. Despite the velocity estimation from the non-filtered EPOD (Fig. 4a) has reasonable levels of error, the pressure estimation (Fig. 4c) is affected by a large estimation error. The error on the pressure is mainly produced by the time derivative of the velocity (Fig. 4b), due to the amplification of the small estimation errors in the POD time coefficients by the time differentiation. Similar levels of error are present in the pressure estimation from the TH (Fig. 4k), also in this case produced by large errors in the time derivative (Fig. 4j). The $\Xi$-filtered EPOD, instead, produces slightly worse results in terms of estimated velocity fields (Fig. 4d), but shows much lower errors on the time derivative (Fig. 4e) and, thus, on the pressure estimation (Fig. 4f). The results of the $\psi$-filtered EPOD shows, at least for the present dataset, the lowest levels of error both in term of velocity field estimation (Fig. $4 \mathrm{~g}$ ) and of its time derivative (Fig. 4h), which lead to very low errors in the pressure estimation (Fig. 4i).

It is worth to remark that the EPOD estimation, differently from the TH, provides by default a set of timeresolved velocity fields, thus can be employed to estimate the history of velocity and pressure fluctuations, which can be employed in time-dependent studies. Taylor's hypothesis, by contrast, is generally employed to estimate the time-derivative in a measured flow field snapshot, even if it can be used to propagate the velocity field as explained above. To clarify how the propagation of TH will accumulate noise, the RMS error of the estimated pressure in the whole domain is plotted in Fig. 5a over 60 frames (which corresponds to 1 convection time for the main flow) from the beginning of propagation. The results are compared both to the TH-based pressure estimation without propagation, i.e. assuming that the exact velocity field is known at each frame, and to the EPOD estimation. The $\psi$-filtered EPOD estimation is the most accurate method to reconstruct time-resolved flow and pressure field series, keeping the least error all the time. The error of TH without propagation has higher levels of error that the EPOD, but is keeping stable around a fixed value through time. The error of TH with propagation is similar for the first 10 frames and starts to grow 


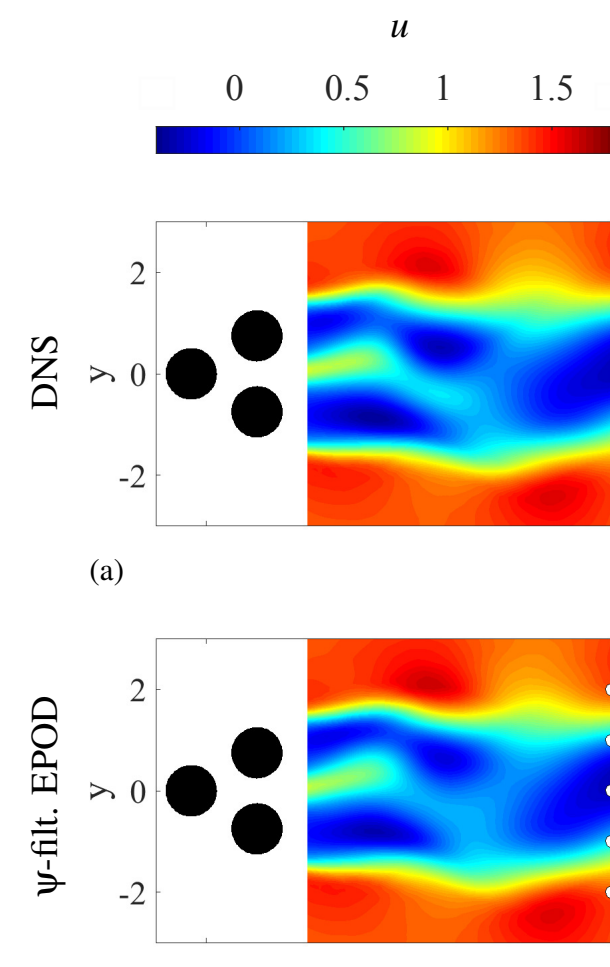

(d)

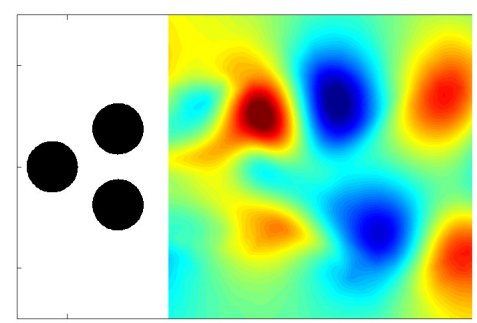

(b)

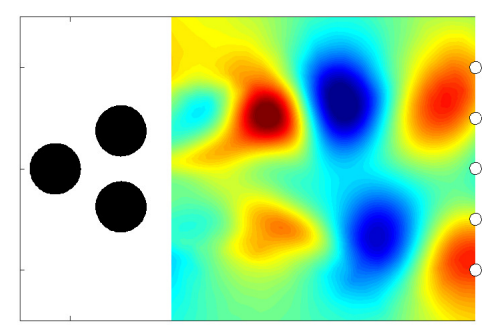

(e)

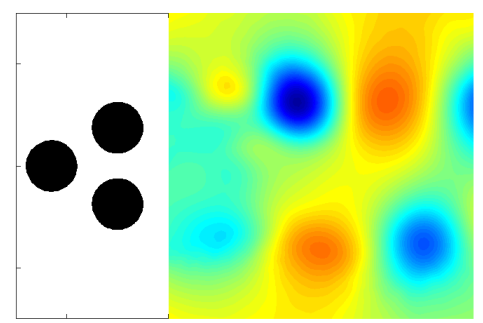

(c)

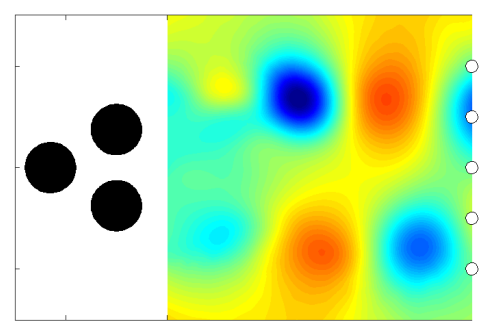

(f)

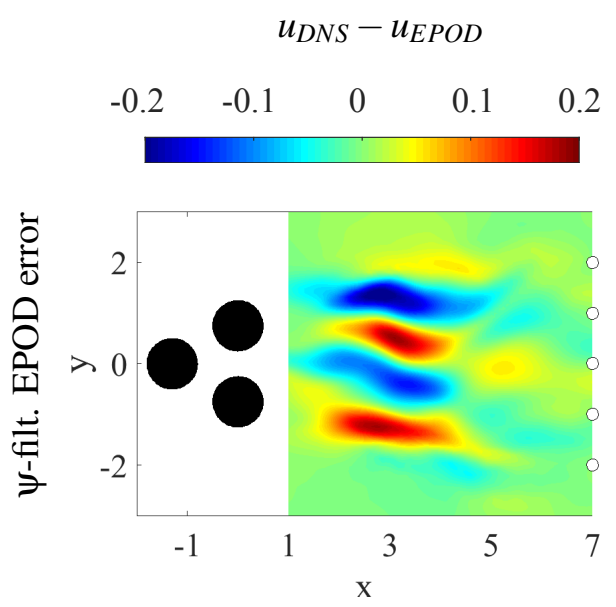

(g)

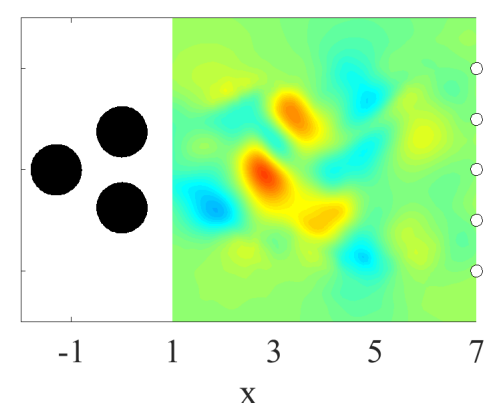

(h)

$$
v_{D N S}-v_{E P O D} \quad p_{D N S}-p_{E P O D}
$$
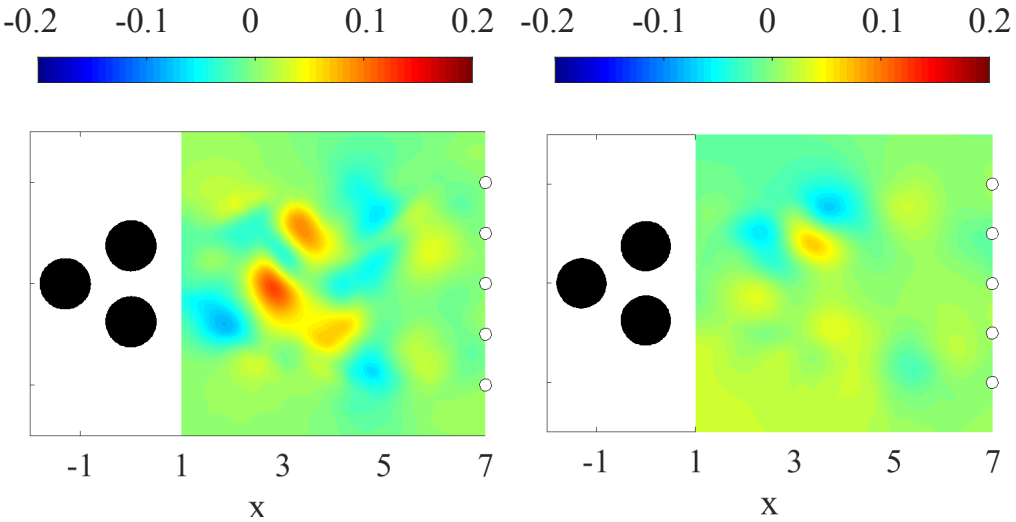

(i)

Figure 3: Estimated and exact velocity and pressure fields for a single snapshot: (a) streamwise velocity from DNS; (b) crosswise velocity from DNS; (c) pressure from DNS; (d) streamwise velocity from EPOD; (e) crosswise velocity from EPOD; (f) pressure from EPOD; (g) EPOD estimation error on streamwise velocity; (h) EPOD estimation error on crosswise velocity; (i) EPOD estimation error on pressure. 


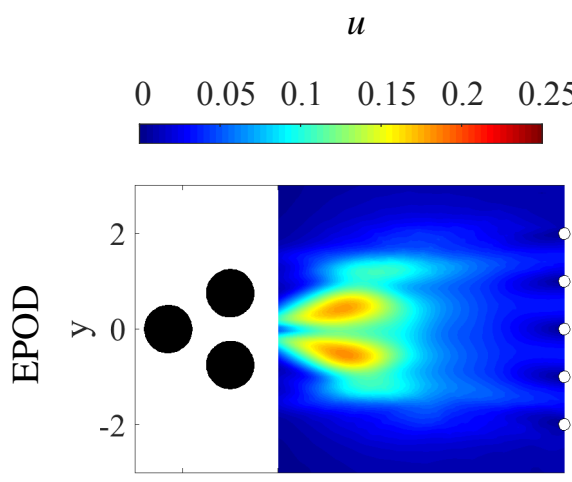

$d u / d t$

$p$

(a)

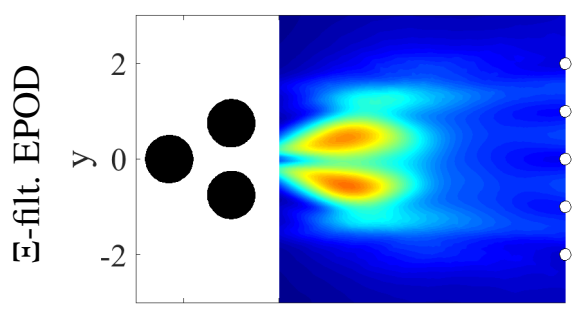

(d)

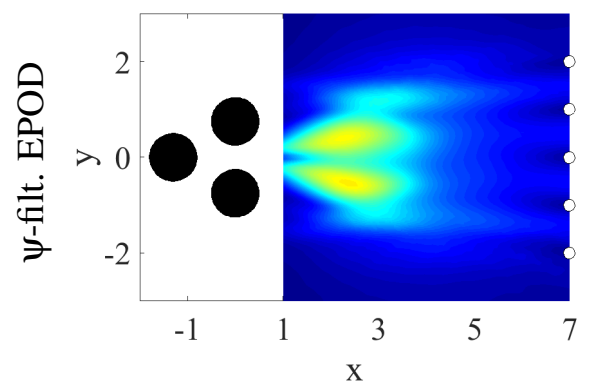

(g)

E

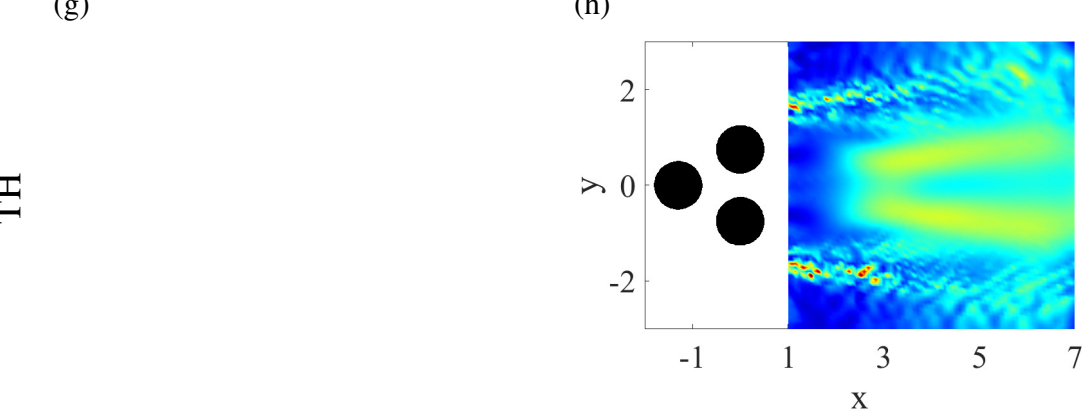

(j)

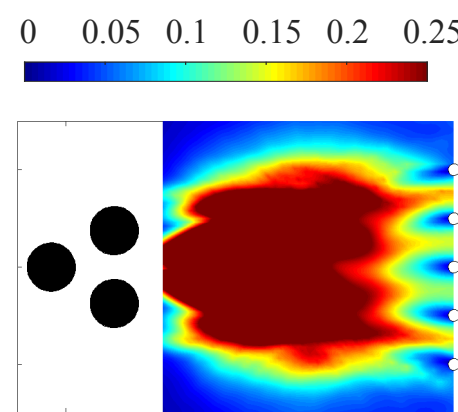

(b)

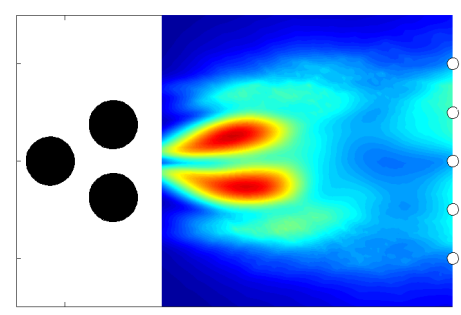

(e)

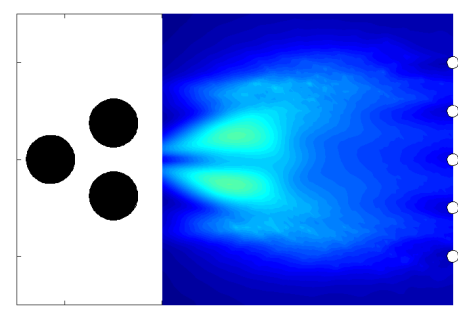

(h)

(k)
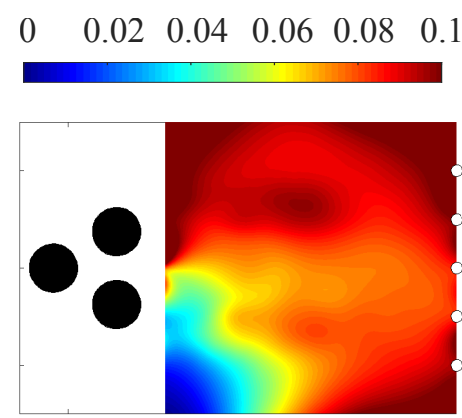

(c)

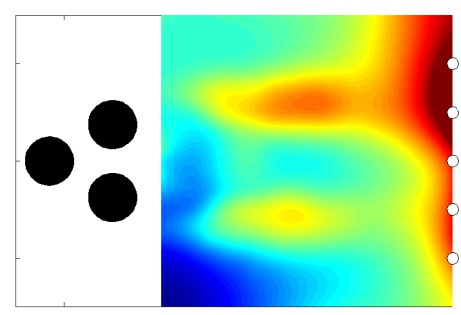

(f)

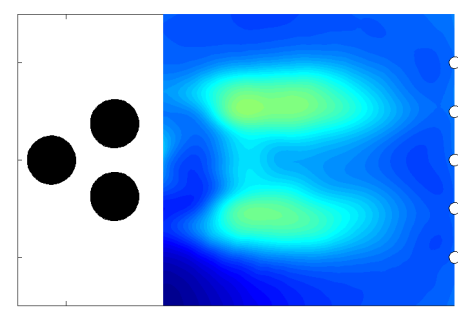

(i)

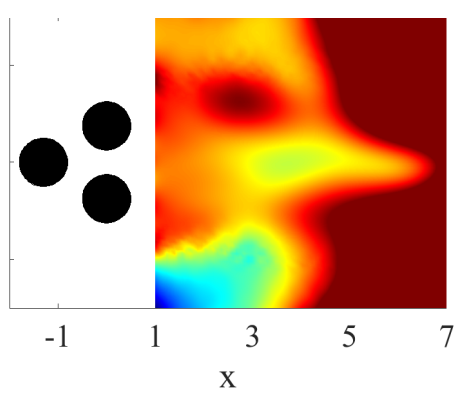

Figure 4: RMS error map of velocity and pressure fields estimation using the EPOD without filter, the $\Xi$-filtered EPOD, the $\Psi$-filtered EPOD and the Taylor's hypothesis. The first column shows error on the streamwise velocity, the second column shows the one on the time derivative of streamwise velocity, and the last column shows the error on pressure. 


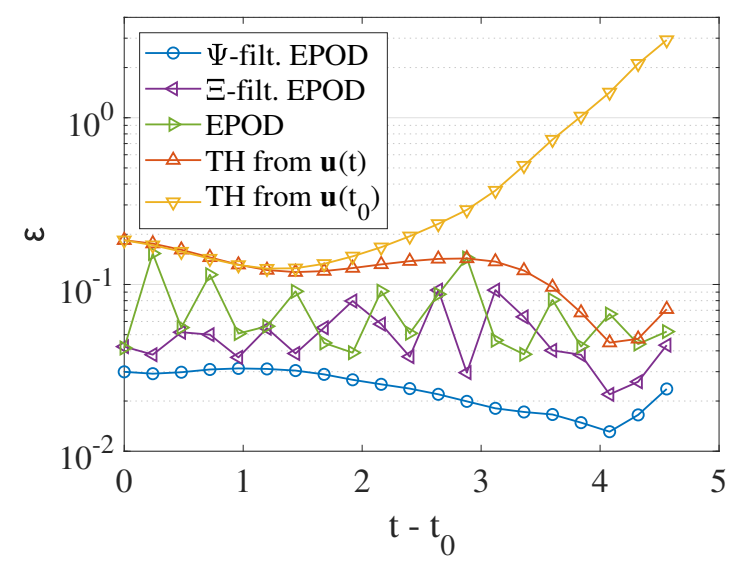

(a)

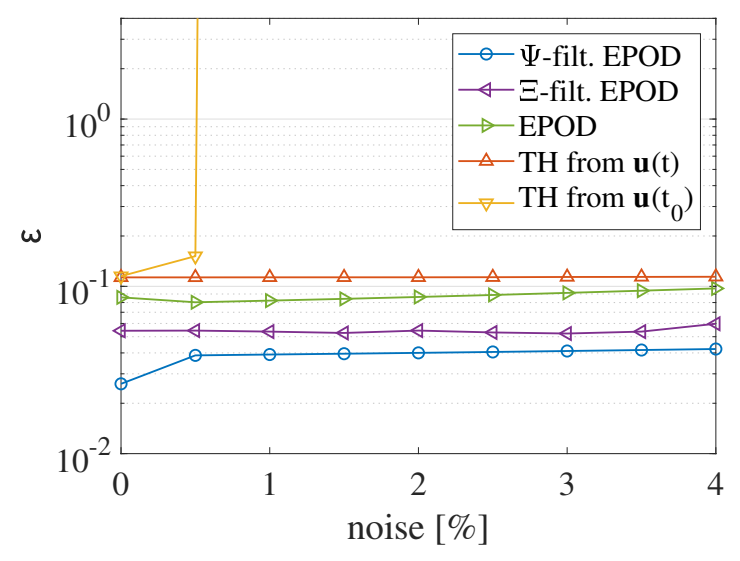

(b)

Figure 5: (a) RMS error of estimated pressure over the whole domain as propagates in time after the measured frame. (b) RMS error of estimated pressure over the whole domain and for 10 snapshots propagation for different levels of noise in training data. $\psi$-filtered EPOD (blue circles), $\Xi$-filtered EPOD (purple leftward triangles), un-filtered EPOD (green rightward triangles), non-propagated TH (red upward triangles) and time-propagated TH (yellow downward triangles).

quickly after that point. In Fig. 5b, the RMS error of estimated pressure in the whole domain and for the first 10 snapshots after the measured one is reported for different levels of noise in the training data and probe measurement in testing data using the same methods as in Fig. 5a. Gaussian noise with zero mean value and standard error up to $4 \%$ of the freestream velocity is used. It shows that the $\psi$-filtered EPOD estimation has significant less error in the pressure estimation than $\mathrm{TH}$ (whether propagation is used or not) for all the noise levels tested. By contrast, the propagated TH error explodes as soon as the noise level is higher than $0.5 \%$ of the freestream velocity.

\subsection{Channel flow}

The EPOD estimation is validated using a second synthetic dataset extracted from the DNS of a channel flow contained the in Johns Hopkins Turbulence Databases (Li et al., 2008; Yu et al., 2012). The DNS is solved in a domain of size $8 \pi \times 2 \times 3 \pi$, using $2048 \times 512 \times 1536$ nodes, at friction-velocity-based Reynolds number $R e_{\tau} \approx 1000$, and the time interval in storage is 0.065 . The EPOD estimation is evaluated in a training dataset composed by sub-domain of size $1 \times 1$ with a time interval of $2 / 3$ of the turnover time, thus being non-time-resolved. Additionally, the dataset includes 10 probes measuring the 2 in-plane components of velocity placed along the downstream boundary $(x=1)$ of the sub-domain spaced of 0.1 in the $y$ direction and recording 152 samples for each snapshot with a time-spacing of 0.065 , which corresponds to one subdomain turnover time. To collect enough independent snapshots, the sub-domains are extracted from different positions exploiting statistical homogeneity, similarly as to what reported in (Discetti et al., 2018).

The RMS error map of pressure using the $\Xi$-filtered EPOD estimation and the Taylor's hypothesis (both with and without propagation) are shown in Fig. 6. The EPOD for this case is trained over 6400 snapshots. The testing dataset, instead, is composed by 240 time-resolved frames. Unlike the fluidic pinball case, the un-propagated Taylor's hypothesis (Fig.6b) performs better than the EPOD estimation (Fig.6a), especially in the near wall region $(0.7<y<1)$ and in the upstream boundary of the domain $(0<x<0.1)$. This higher error might be explained by several reasons. Firstly, the channel flow reported here is characterized by a moderate Reynolds number, thus being completely turbulent and having a much larger wealth of turbulent scales (generally recovered by a larger wealth of POD modes), lowering the correlation between field and probe modes. Secondly, larger turbulence introduces stronger three-dimensionality in the flow, thus further reducing the correlation level. Thirdly, the channel is characterized by much lower convection velocity in the near-wall region. This means that in the temporal span recorded by the fast probes (roughly one turnover time, i.e. the time required by a fluid particle to span the entire sub-domain when convected at the centerline velocity) is not long enough to sense all the fluid structures passing. Finally, the near-wall region is characterized by a stronger deformation of the small-scale fluid structures as well as by their interaction with 


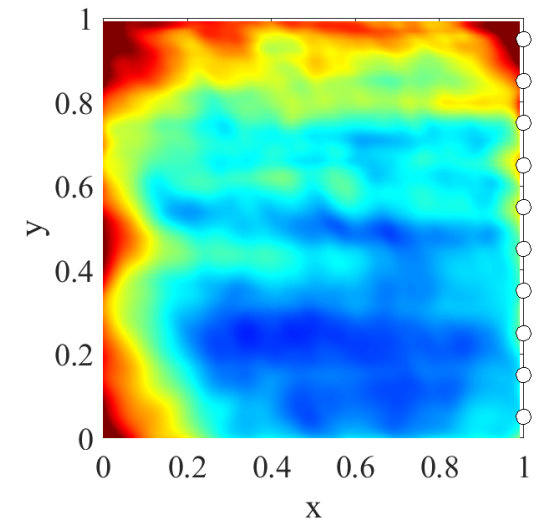

(a)

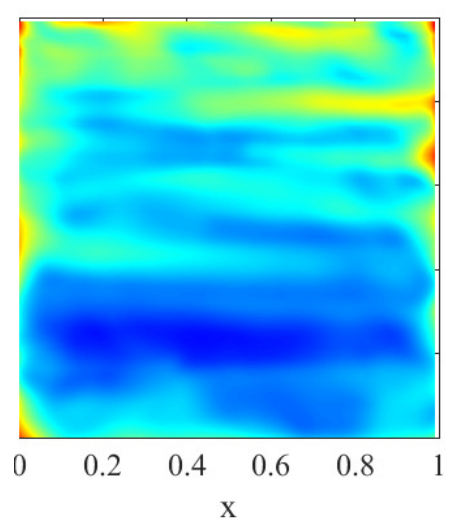

(b)

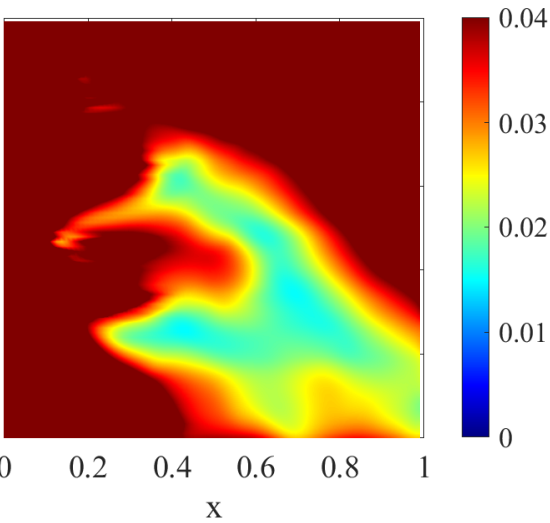

(c)

Figure 6: RMS error map of velocity and pressure fields estimation using (a) $\Xi$-filtered EPOD estimation, (b) the un-propagated Taylor's hypothesis, (c) time-propagated Taylor's hypothesis.

larger structures, meaning that the correlation between the field modes and probe modes is lower. Despite this, the EPOD estimation offers reasonably good results in terms of pressure (Fig.6a). Additionally, EPOD estimation offers an inherently advantage with respect to the TH in that it provides time-series for tracking the fluctuations of pressure and velocity in time. While this can be achieved also by the TH by propagating in time the velocity fields, the results of such estimation contains much higher levels of noise (Fig.6c).

Fig. 7a compares the EPOD estimation and the Taylor's hypothesis in recovering time-series out of an initial snapshot in terms of RMS pressure estimation error over the sub-domain. As already commented, in the channel flow, the TH-based estimation with no time-propagation of the flow field is superior to the EPOD approach. However, when the TH is used also to propagate the velocity field from a single snapshot, the error increases quickly with time. On the other hand, the EPOD approach mantains stable levels of error through all the time sequence, producing better results than the TH after 20 steps. Fig. $7 \mathrm{~b}$ reports the RMS error of the pressure in the whole sub-domain and over the first 10 snapshot of propagation with different levels of noise on the velocity field data. The non-propagated TH proves to be the most robust one to noise. EPOD, independently for the filtering method, has rather constant error independently from the noise level (the unfiltered EPOD has only slightly increasing noise). The TH with propagation, instead, has an error which increases with the noise, even if the propagation is limited to 10 frames, proving to provide the least robust pressure time-series estimation.

\section{Summary}

A novel approach to estimate instantaneous pressure fields using snapshot PIV (i.e. without time resolution) has been presented. The method is based on using synchronized field measurements from PIV and point measurements using high-repetition rate probes (such as hot-wires or pressure transducers, among others). The field are then estimated at the same time resolution of the probes using extended POD, and the pressure gradient is extracted from the Navier-Stokes equations and integrated in space. The method has demonstrated to be sufficiently accurate to perform this task, and superior to Taylor's hypothesis if the POD reconstruction is sufficiently compact, and provided that some filtering is applied to reduce time fluctuations. The proposed approach has also the advantage of having error independent on the time when PIV snapshots have been adquired, and indeed it only needs probe measurements once the training data have been captured. This opens the path to reconstruct pressure fields not only corresponding to the instants when PIV fields have been captured, but also in-between snapshots, or in general in instants when PIV is not available (for example, for a separate probe data sequence capture outside of the training dataset).

\section{Acknowledgements}

This project has received funding from the European Research Council (ERC) under the European Union's Horizon 2020 research and innovation programme (grant agreement No 949085). This document reflects only the author's view 


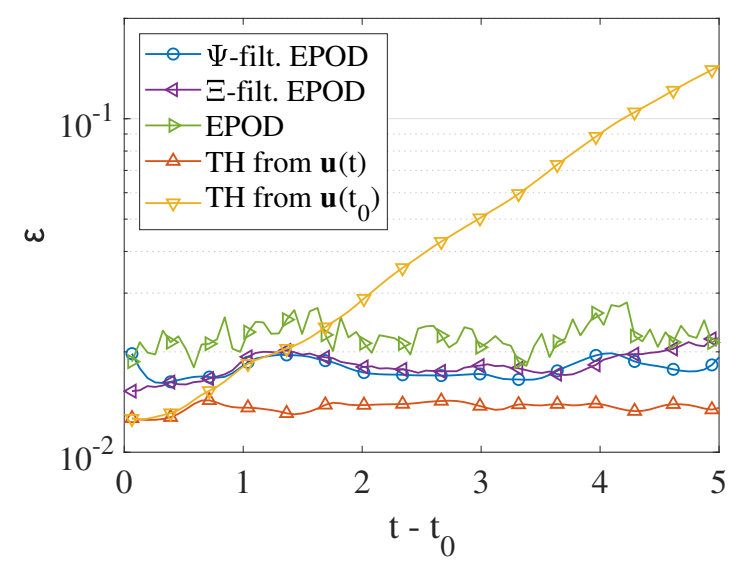

(a)

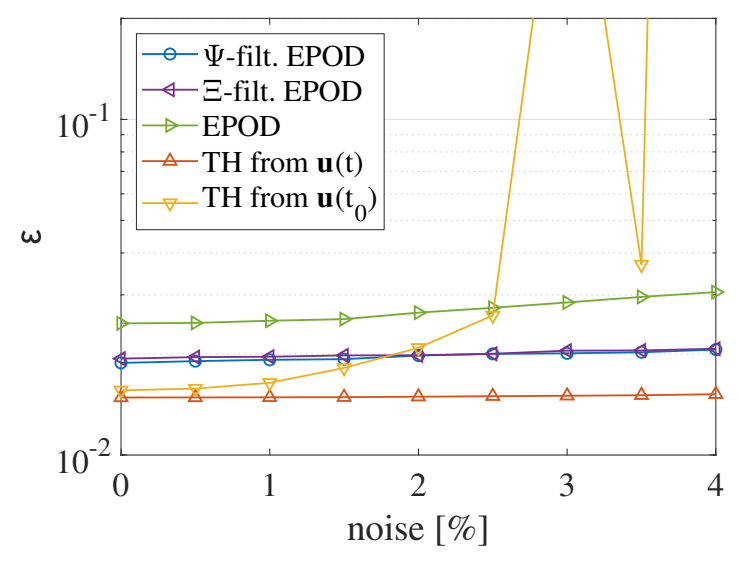

(b)

Figure 7: (a) RMS error of estimated pressure over the whole domain as propagates in time after the measured frame. (b) RMS error of estimated pressure over the whole domain and for 10 snapshots propagation for different levels of noise in training data. $\psi$-filtered EPOD (blue circles), $\Xi$-filtered EPOD (purple leftward triangles), un-filtered EPOD (green rightward triangles), non-propagated TH (red upward triangles) and time-propagated TH (yellow downward triangles).

and the Agency and the Commission are not responsible for any use that may be made of the information it contains.

\section{References}

Borée J (2003) Extended proper orthogonal decomposition: a tool to analyse correlated events in turbulent flows. Experiments in fluids 35:188-192

Deng N, Noack BR, Morzyński M, and Pastur LR (2020) Low-order model for successive bifurcations of the fluidic pinball. Journal of Fluid Mechanics 884

Discetti S, Bellani G, Örlü R, Serpieri J, Vila CS, Raiola M, Zheng X, Mascotelli L, Talamelli A, and Ianiro A (2019) Characterization of very-large-scale motions in high-Re pipe flows. Experimental Thermal and Fluid Science 104:1-8

Discetti S, Raiola M, and Ianiro A (2018) Estimation of time-resolved turbulent fields through correlation of non-time-resolved field measurements and time-resolved point measurements. Experimental Thermal and Fluid Science 93:119-130

Hosseini Z, Martinuzzi RJ, and Noack BR (2015) Sensor-based estimation of the velocity in the wake of a low-aspect-ratio pyramid. Experiments in Fluids 56:13

Li Y, Perlman E, Wan M, Yang Y, Meneveau C, Burns R, Chen S, Szalay A, and Eyink G (2008) A public turbulence database cluster and applications to study lagrangian evolution of velocity increments in turbulence. Journal of Turbulence 9:N31

Richardson LF (1910) The approximate arithmetical solution by finite differences of physical problems involving differential equations, with an application to the stresses in a masonry dam. Philosophical Transactions of the Royal Society A 210:307

Schneiders JF, Avallone F, Pröbsting S, Ragni D, and Scarano F (2018) Pressure spectra from single-snapshot tomographic PIV. Experiments in Fluids 59:57

Sicot C, Perrin R, Tran T, and Borée J (2012) Wall pressure and conditional flow structures downstream of a reattaching flow region. International Journal of Heat and Fluid Flow 35:119-129

Tinney C, Ukeiley L, and Glauser MN (2008) Low-dimensional characteristics of a transonic jet. part 2. estimate and far-field prediction. Journal of Fluid Mechanics 615:53-92

Tronchin T, David L, and Farcy A (2015) Loads and pressure evaluation of the flow around a flapping wing from instantaneous 3d velocity measurements. Experiments in Fluids 56

Van der Kindere J, Laskari A, Ganapathisubramani B, and De Kat R (2019) Pressure from 2D snapshot PIV. Experiments in Fluids 60:32

Van Gent P, Michaelis D, Van Oudheusden B, Weiss PÉ, de Kat R, Laskari A, Jeon YJ, David L, Schanz D, Huhn F et al. (2017) Comparative assessment of pressure field reconstructions from particle image velocimetry measurements and lagrangian particle tracking. Experiments in Fluids 58:33

Van Oudheusden B (2013) PIV-based pressure measurement. Measurement Science and Technology 24:032001

Yu H, Kanov K, Perlman E, Graham J, Frederix E, Burns R, Szalay A, Eyink G, and Meneveau C (2012) Studying lagrangian dynamics of turbulence using on-demand fluid particle tracking in a public turbulence database. Journal of Turbulence 13:N12 